\title{
RESCATES BIOARQUEOLÓGICOS EN EL VALLE MEDIO DEL RÍO NEGRO (PROVINCIA DE RÍO NEGRO): ELPOTENCIALINFORMATIVO DEL REGISTRO ALTAMENTE PERTURBADO
}

\author{
BIOARCHAEOLOGICAL RESCUES IN THE MID VALLEY OF THE NEGRO RIVER \\ (PROVINCE OF RÍO NEGRO): THE INFORMATIVE POTENTIAL OF HIGHLY \\ DISTURBED RECORD
}

\author{
Alejandro Serna ${ }^{1,2 *}$ y Victoria Romano ${ }^{2}$ \\ ${ }^{I}$ Consejo Nacional Científico y Tecnológico (CONICET). La Plata. Argentina \\ ${ }^{2}$ División Arqueología, Facultad de Ciencias Naturales y Museo. Universidad Nacional de La Plata. La Plata. Argentina
}

PALABRAS CLAVE restos humanos; tafonomía; cuantificación; análisis sexo-etario; modificación craneana

\begin{abstract}
RESUMEN Este trabajo se centra en el análisis de tres conjuntos bioarqueológicos altamente perturbados por la acción antrópica moderna, rescatados en el valle del río Negro (provincia de Río Negro). Su objetivo es dar a conocer los resultados del análisis cuantitativo, sexo-etario y tafonómico realizado sobre los conjuntos óseos recuperados en los sitios Fábrega, Negro Muerto 3 y Loma de los Muertos y evaluar el impacto de la incorporación de este registro a la discusión a nivel intra-sitio y regional. Los resultados de este trabajo permitieron incorporar un
\end{abstract}

nuevo sitio con evidencias inhumatorias al esquema regional, ampliar y ajustar el número de individuos y espectro etario en otros sitios, además de incorporar información novedosa acerca de prácticas de modificación craneana y procesamiento de cadáveres. Los nuevos datos generados demuestran el potencial informativo, en términos paleodemográficos y culturales, del registro bioarqueológico altamente impactado por la acción antrópica moderna. Rev Arg Antrop Biol 20(2), 2018. doi:10.17139/ raab.2018.0020.02.03

\section{KEY WORDS human remains; taphonomy; quantification; sex-age analysis; cranial modification}

ABSTRACT This work focuses on the analysis of three bioarchaeological samples highly disturbed by modern anthropic action, which were recovered from the Negro river valley (province of Río Negro). Our objective is to present the results of the quantitative, sex-age, and taphonomic analyses carried out on the remains recovered in Fábrega, Negro Muerto 3, and Loma de los Muertos sites, and to assess the impact of incorporating this kind of disturbed samples into the intra-site and regional discussions. The

El registro bioarqueológico del noreste patagónico se presenta de distintas formas (i.e. entierros primarios y secundarios, simples y múltiples, pintados, asociados a materiales culturales y faunísticos) y se caracteriza por su elevada frecuencia (Bórmida, 1950; Del Papa et al., 2011; Favier Dubois, Borella y Tykot, 2009; Martínez, 2010; Moreno, 1874; Outes, 1926; Prates y Di Prado, 2013; Peronja, Sánchez y Juliá, 1987; Vignati, 1931). Durante los últimos diez años, ha habido un incremento en la demanda de pedidos de relevamiento y rescate arqueológico vinculado con la aparición de restos esqueletales, particularmente en el área del valle medio e inferior del río Negro. Como consecuencia de estos pedidos se han detectado e iniciado acciones de rescate sobre numerosos sitios: Loma results allowed the incorporation of a new mortuary site to the regional scheme, the expansion and adjustment of the number of individuals and age spectrum in other sites, and the incorporation of new information about cranial modification and post-mortem processing. The new data generated here demonstrates the informative potential of bioarchaeological record highly impacted by modern action. Rev Arg Antrop Biol 20(2), 2018. doi:10.17139/ raab.2018.0020.02.03

de los Muertos -LM- (Prates, Di Prado, Mange y Serna, 2010; Prates, Flensborg y Bayala, 2010), La Victoria 5 -LV5- (Prates, Luchsinger, Scabuzzo y Mansegosa, 2011); Negro Muerto 2 -NM2- (Serna y Prates, 2012); Negro Muerto 3 -NM3- (Mange, Prates, González Venanzi y Di Lorenzo, 2016; Serna, 2014); Barrio Cooperativa 8 de Diciembre -BC8D- (Prates y Serna,

Financiamiento: ANPCyT (PICT 2015-3645).

*Correspondencia a: Alejandro Serna. Unidad de Investigación Anexo Museo. Laboratorio N 128. Calle 60 y 122. 1900 La Plata. Buenos Aires. Argentina.

E-mail: aserna@fcnym.unlp.edu.ar

Recibido 3 Marzo 2017; aceptado 20 Octubre 2017

doi:10.17139/raab.2018.0020.02.03 
2015), Fábrega -Fa- (E. Mange, comunicación personal, 2017) y Chimpay -Ch- (Prates, Serna, Mange y de Jong, 2016; Serna, Prates y Luna, 2015) (Fig. 1). En la mayor parte de los casos estas solicitudes son el producto de hallazgos accidentales durante el trabajo agropecuario y urbanístico. Así, la actividad antrópica moderna constituye el principal promotor de los hallazgos bioarqueológicos de la región.

De este modo, las mismas acciones que promueven este tipo de hallazgos producen generalmente un gran volumen de registro bioarqueológico deteriorado, desorganizado anatómicamente y descontextualizado arqueológicamente (Borella, Mariano y Favier Dubois, 2007). Conjuntos óseos de estas características suelen considerarse pobres en términos de su potencial como fuente de información y, por consiguiente, relegados o descartados para la investigación. Sin embargo, bajo el adecuado tratamiento analítico este tipo de registro puede convertirse en un referente empírico válido y útil para el desarrollo de investigaciones arqueológicas (Adams y Byrd, 2008; Borella et al., 2007; Knüsel y Outram, 2004; Luna, 2010; Martín, Barberena y Guichón, 2004). El presente trabajo se centra en el registro bioarqueológico altamente per- turbado recuperado durante los rescates de los sitios Fábrega, Negro Muerto 3 y Loma de los Muertos, ubicados en el valle del río Negro. Su objetivo es dar a conocer los resultados del análisis cuantitativo, sexo-etario y tafonómico de los conjuntos óseos rescatados y evaluar el impacto de la incorporación de este registro a la discusión a nivel intra-sitio y regional.

\section{MATERIAL Y MÉTODOS}

\section{Las muestras analizadas}

Los conjuntos óseos analizados en este trabajo provienen de rescates llevados a cabo en los sitios: Fábrega (Fa); Negro Muerto 3 (NM3) y Loma de los Muertos (LM) (Fig. 1). El sitio Fa se halla sobre un médano ubicado en una chacra localizada en cercanías de la localidad de Lamarque (39²6'57's; 65³9'49'O). Se hallaron restos humanos semi-enterrados y en estratigrafía, además de escasos restos faunísticos y líticos. Por el momento no se dispone de una cronología absoluta para el sitio (E. Mange, comunicación personal 2016). El sitio NM3 forma parte de la localidad arqueológica Negro Muerto y está ubicado en la margen norte del río Negro

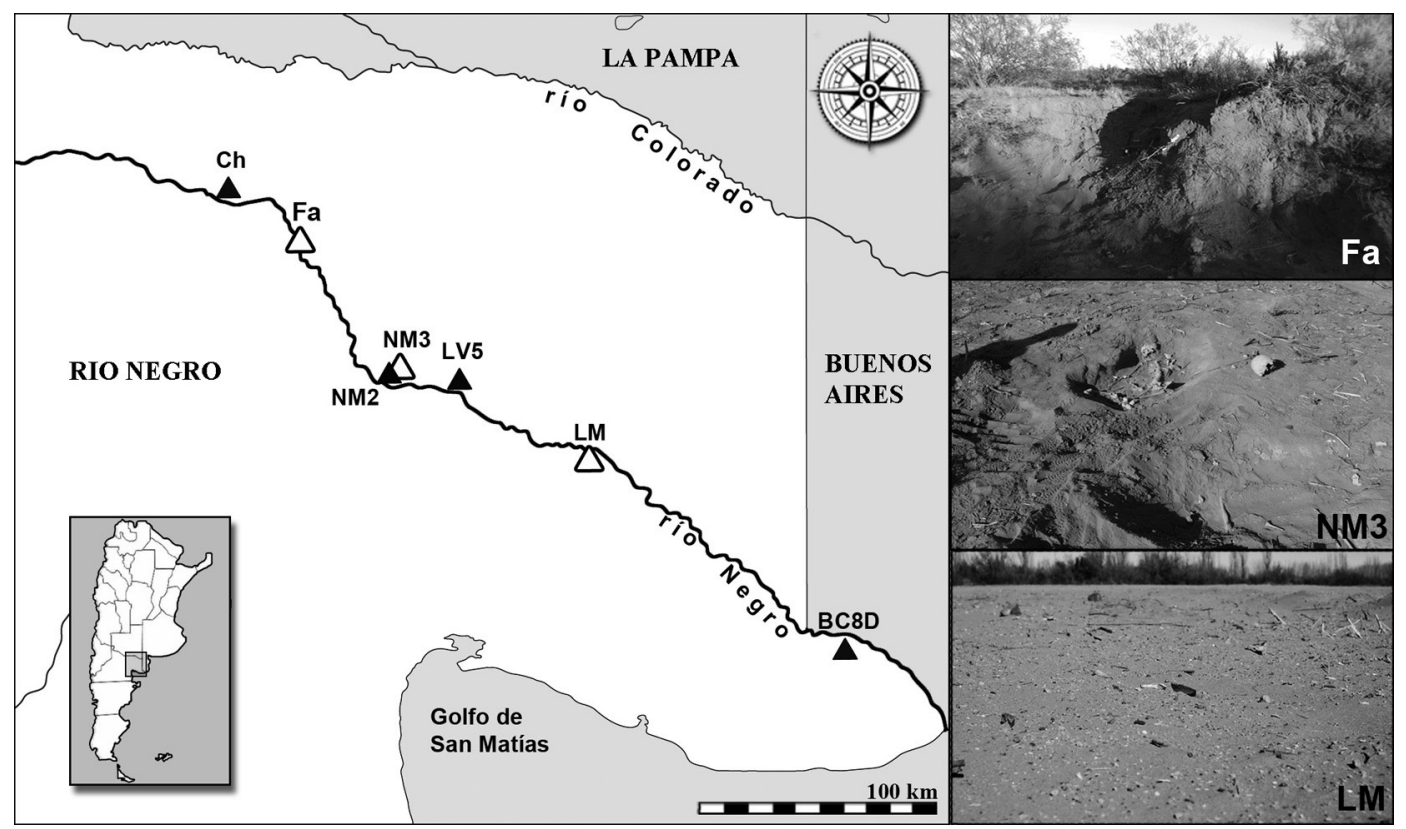

Fig. 1. Mapa con la ubicación de los sitios bioarqueológicos rescatados durante la última década (en color blanco los sitios analizados en este trabajo). 
sobre una pequeña elevación de matriz arenosa contigua a un canal de inundación (3949'0.4'S; $\left.65^{\circ} 17^{\prime} 2^{\prime} \mathrm{O}\right)$. Además de los restos humanos superficiales y altamente perturbados en un sentido espacial y anatómico, se hallaron entierros primarios in situ (los cuales no se incluyen en este análisis), materiales faunísticos y líticos, alfarería y estructuras de combustión constituidas por sedimentos compactados. Dos fechados radiocarbónicos de $c a .1400$ a 1100 años AP realizados sobre material arqueofaunístico permiten asignar el sitio al Holoceno tardío final (Mange et al., 2016). El sitio LM se encuentra emplazado sobre una formación de arena sobreelevada en la margen sur del río Negro, en cercanías de la ciudad de General Conesa $\left(40^{\circ} 08^{\prime} 47^{\prime}\right.$ 'S; 64'16'19'O). Se rescataron restos humanos superficiales, entierros primarios in situ (Prates et al., 2010b), material arqueofaunístico, lítico y cerámico, pigmentos minerales y elementos ornamentales elaborados sobre valva y sobre hueso. Una serie de fechados radiocarbónicos realizados sobre restos humanos indica una cronología de $c a$. 3.000 a 2.000 años AP, la cual ubica al sitio en el Holoceno tardío inicial (Prates et al., 2010a, b).

\section{Metodología}

Los restos bioarqueológicos recuperados en cada sitio fueron acondicionados, inventariados y procesados cuantitativamente. Se cuantificó el número de especímenes óseos (NISP), se calculó el número mínimo de elementos (NME), se determinó el número mínimo de individuos (NMI), la abundancia relativa de partes esqueletarias (MAU y MAU\%) y el índice de supervivencia (ISup.) (Grayson, 1984; Gutiérrez, 2004; Lyman, 2008). La determinación sexual se efectuó sobre los elementos que mejor expresan el dimorfismo, tales como el coxal, la mandíbula y el cráneo (Buikstra y Ubelaker, 1994; Luna y Aranda, 2005; Schutkowski, 1993). La estimación de la edad también se realizó a nivel de elemento y se consideraron los grados de fusión ósea, la erupción dentaria, las características morfológicas generales y métricas (Ubelaker, 1989; Scheuer y Black, 2000; White y Folkens, 2005). Los elementos óseos fueron divididos en las categorías etarias propuestas por Buikstra y Ubelaker (1994): infante (0-3 años), niño (3-12 años), adolescente (12-20 años) y adulto ( $>20$ años). Además, se relevó la acción de distintos procesos y agentes tafonómicos tanto naturales como antrópicos (Lyman, 1994, 2010). Como indicadores de preservación, se tuvieron en cuenta la presencia de fracturas postdepositacionales y la completitud de cada elemento dividiéndola en 4 categorías ordinales (categoría 1 -mala-: 0-24,9\%; categoría 2 -regular-: 25-49,9\%; categoría 3 -buena-: 50-74,9\%; categoría 4 -muy buena-: 75-100\%) basadas en los criterios de Buikstra y Ubelaker (1994). Como medida del grado de exposición superficial a la que pudieron haber estado sometidos los especímenes óseos se evaluó la meteorización utilizando los estadios propuestos por Behrensmeyer (1978) y la presencia de blanqueamiento ("sun bleaching”) debido a la radiación solar (Dupras y Schultz, 2013). Además, sobre este último, se consignaron los casos con exposición diferencial, es decir aquellos casos en los cuales un sector del espécimen está marcadamente afectado y el otro no (Guichón, Muñoz y Borrero, 2000). Otras variables consideradas para relevar y caracterizar su efecto sobre la superficie ósea fueron: marcas de raíces y roedores, depositaciones químicas (e.g. carbonato de calcio y óxido de manganeso), huellas de corte de origen antrópico y deterioro químico (Binford, 1981; Gutiérrez, 2004; Lyman, 1994). Finalmente, en los casos que fue posible ensamblar cráneos a partir de los especímenes óseos, se observaron escópicamente en busca de señales de modificaciones morfológicas culturales (Dembo e Imbelloni, 1938).

\section{RESULTADOS}

\section{Sitio Fábrega}

El sitio Fa cuenta con un NISP $=86$, que conforma un $\mathrm{NME}=46$ y un $\mathrm{MNI}=3$ derivado de los cráneos (Tabla 1; véase mayor detalle en Material Suplementario 1). En general los elementos con mayor representación son el húmero y el cráneo, mientras que los que conforman el antebrazo, la columna vertebral y los miembros inferiores son los menos representados (Fig. 2). Los análisis sexo-etarios indican que hay, al menos, un individuo de sexo masculino y otro femenino, $\mathrm{y}$ que todos los elementos recuperados corres- 


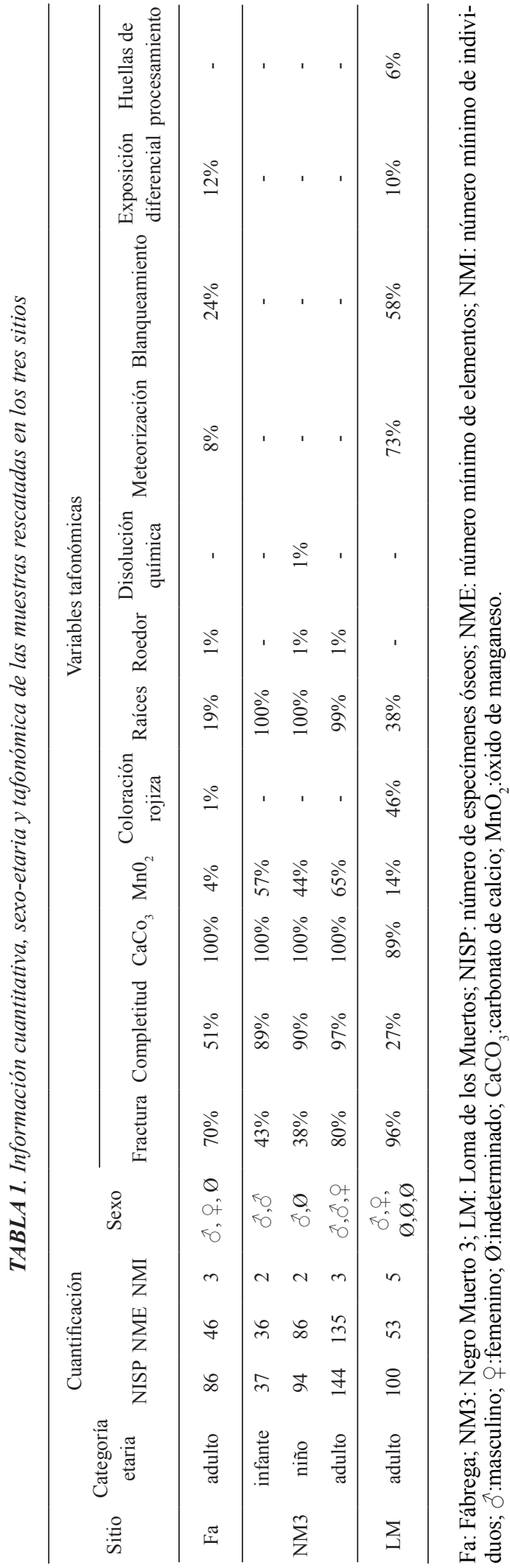

ponden a adultos ( $>20$ años). Respecto de la preservación, aunque más de la mitad de la muestra se encuentra fracturada postdepositacionalmente (70\%), un $51 \%$ de los elementos que la componen presenta una completitud buena a muy buena ( $\geq 50 \%$ ). Respecto de las depositaciones químicas, el 100\% de la muestra presenta rastros de carbonato de calcio en forma de una delgada película y un $4 \%$ exhibe un moteado oscuro debido al óxido de manganeso. Además, se registró una tinción rosada a rojiza en el borde interior de una fractura de un fragmento de cráneo (Fig. 3a). Otros agentes que afectaron la superficie de los huesos fueron las raíces en un 19\% dejando marcas superficiales de tipo dendrítico, y un 1\% por marcas de roedores. Un $8 \%$ de los restos presenta signos leves de meteorización y un $24 \%$ de blanqueamiento, de los cuales un 12\% exhibe exposición diferencial (Tabla 1).

\section{Sitio Negro Muerto 3}

El conjunto de NM3 cuenta con un $\mathrm{NISP}=275$, que representa un $\mathrm{NME}=257$ y un $\mathrm{NMI}=7$ derivado principalmente de huesos largos y coxales (Tabla 1; véase mayor detalle en Material Suplementario 2). A través de la estimación de la edad se identificaron tres categorías: infante, niño y adulto. El conjunto infante presenta un $\mathrm{NME}=36$ y un $\mathrm{NMI}=2$ (un individuo de 1,5 a 2,5 años y otro de 2,5 a 3,5 años) calculado a partir de los ilion, los cuales también indican que ambos individuos son de sexo masculino (Tabla 1). El conjunto niño presenta un $\mathrm{NME}=86$, un $\mathrm{NMI}=2$ (un individuo de 3,5 a 5,5 años y otro de 9,5 a 10,5 años) derivado de los fémures, y la determinación sexual muestra que al menos un individuo es masculino. El conjunto adulto consta de un $\mathrm{NME}=135$ y $\mathrm{NMI}=3$ calculado en base a los fémures (Tabla 1). Además de éstos, los elementos más representados son los radios, las tibias y las escápulas, mientras que los menos son el cráneo, las vértebras cervicales, los metacarpos y metatarsos (Fig. 2). La determinación sexual sobre el conjunto indica la presencia de dos individuos masculinos y uno femenino (Tabla 1). Además, el estudio comparativo de este conjunto y de los entierros primarios hallados en el sitio (Serna, 2014), permitió el remontaje de tres especímenes óseos con distintos elementos de uno de los indivi- 

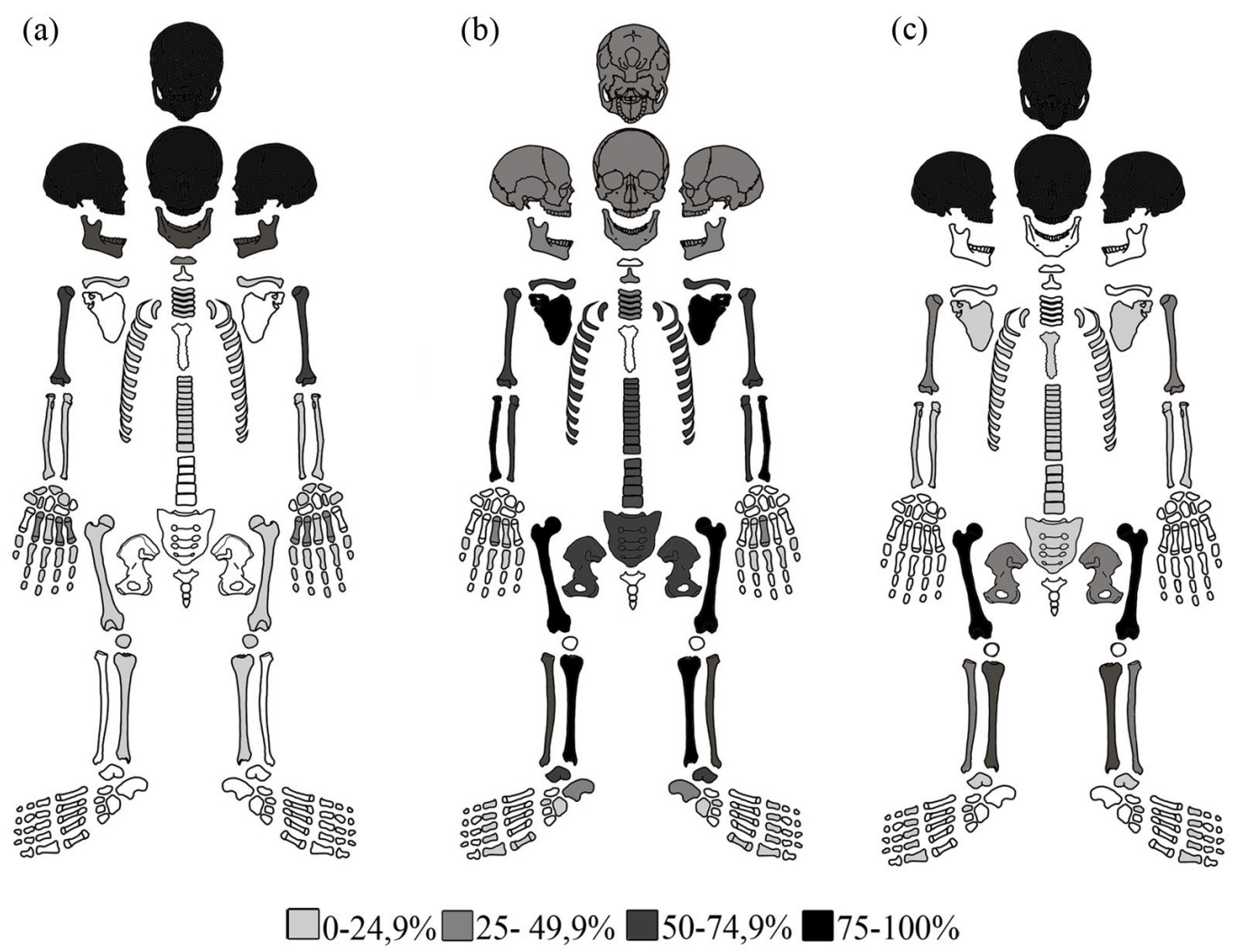

Fig. 2. Representación de los porcentajes de unidades anatómicas en las tres muestras estudiadas: a) Fa; b) NM3 (conjunto adulto) y c) LM.

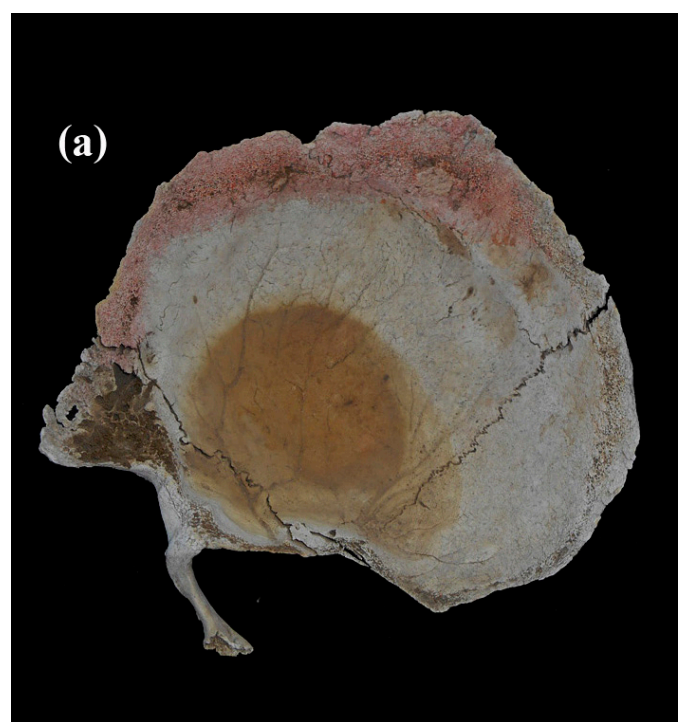

Fig. 3. a) Fragmento de cráneo recuperado en Fa con exposición diferencial y coloración rojiza cubriendo parte de la superficie de fractura. duos hallado en estratigrafía. Con respecto a la preservación de los restos, los porcentajes de fractura postdepositacional son similares y cercanos al $40 \%$ entre los conjuntos infante y niño, mientras que los valores para adulto son considerablemente mayores $(80 \%)$. Pese a estas diferencias, en líneas generales los tres conjuntos presentaron un porcentaje muy elevado $(\geq 89 \%)$ de buena a muy buena completitud (Tabla 1). En cuanto a las depositaciones químicas, la totalidad de los restos recuperados en NM3 presenta carbonato de calcio en forma de una delgada capa de cobertura no homogénea, a diferencia del óxido de manganeso que se expresa en general como pequeñas motas y cuyo porcentaje oscila entre $40 \%$ y $65 \%$ entre los distintos conjuntos. Se registraron patrones dendríticos de variada intensidad generados por las raíces en prácticamente todos los restos recuperados, sólo el $1 \%$ posee marcas de roedores y un espécimen presenta rastros de deterioro químico (Tabla 1). 


\section{Sitio Loma de los Muertos}

La muestra de LM tiene un NISP $=100$, el cual conforma un $\mathrm{NME}=53$ y un $\mathrm{NMI}=5$ calculado a partir de los fémures (Tabla 1; véase mayor detalle en Material Suplementario 3). Algunos de los huesos largos que conforman los miembros inferiores son los que se encuentran más representados, seguidos en orden decreciente la bóveda craneana, coxales y húmeros (Fig. 2). A partir de la evaluación morfológica esta muestra se identificaron cuatro cráneos: A) parcialmente completo con ausencia de la base, del occipital y temporal derecho (adulto de sexo femenino); B) ensamblado a partir de 6 especímenes y representado por el frontal, el occipital y parte de los parietales (adulto de sexo indeterinado); C) ensamblado a partir de 3 especímenes y representado por el parietal derecho (adulto de sexo indeterminado) y D) ensamblado a partir de 2 especímenes y representado por el parietal derecho (adulto de sexo indeterminado). De ellos, únicamente los cráneos A y B pudieron ser evaluados visualmente en busca de señales de modificación cultural gracias a su mayor completitud (Dembo e Imbelloni, 1938). Ambos muestran una compresión en sentido fronto-occipital pero el cráneo B, además, exhibe rastros leves de un surco detrás de la sutura coronal en torno a bregma. Los análisis sexo-etarios indican que en la muestra hay al menos un individuo masculino y otro femenino, y que todos los elementos corresponden a individuos adultos ( $>20$ años) (Tabla 1). En cuanto a la preservación de la muestra, el 96\% de la de los elementos se encuentra fracturado y sólo un $27 \%$ del total presenta una completitud buena a muy buena ( $\geq 50 \%$ ). Respecto de las depositaciones químicas, el 89\% de los especímenes está cubierto por carbonato de calcio distribuido en forma heterogénea, mientras que un $14 \%$ presenta pequeñas manchas aisladas de óxido de manganeso y un $46 \%$ pequeñas motas de aspecto similar a estas últimas pero de color rosado a rojizo (Fig. $3 b)$. Además, la superficie de un $38 \%$ de los especímenes está afectada por la acción de las raíces, exhibiendo un patrón dendrítico de poca profundidad. Respecto de la exposición de los restos, un $73 \%$ presenta signos de meteorización (70\% estadio 1 y $3 \%$ estadio 2 ) y un $58 \%$ blanqueamiento, de los cuales un 10\% exhibe exposición diferencial (Tabla 1). Finalmente, se registraron huellas de procesamiento antrópico en seis elementos (húmero, radio, peroné, tibia y costilla) (Fig. 4). En líneas generales, estas trazas se localizan en distintos sectores de la diáfisis y metáfisis, y consisten en incisiones paralelas a subparalelas de distintas profundidades. Entre estas marcas, se destacan las huellas profundas presentes en el húmero (Fig. 4a, b).

\section{DISCUSIÓN}

\section{Procesos postdepositacionales}

Uno de los aspectos más destacables de la cuantificación de los conjuntos bioarqueológicos aquí analizados es que en todos ellos la completitud esqueletal es muy baja en general y en relación con el NMI de cada muestra, sobre todo para los huesos que conforman los pies y las manos. Esta situación queda claramente evidenciada observando los resultados cuantitativos, particularmente el MAU\% y los valores de supervivencia de los elementos (véanse Fig. 2 y materiales suplementarios). En base a esto, cabe plantearse el origen natural y/o antrópico de estas ausencias o bajas representaciones óseas. En primer lugar, es posible plantear la intervención de ciertos animales como agentes capaces de desplazar y disgregar el material óseo de modo tal que al momento de su recolección se registren varias ausencias. Se ha observado que la acción de los carnívoros, además de alterar la superficie cortical de los huesos, puede alterar la distribución de los restos por medio del transporte y la acumulación (Binford,

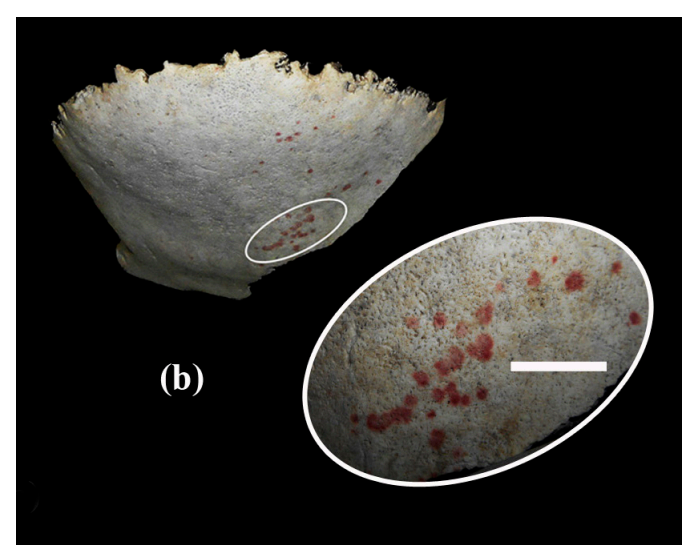

Fig. 3. b) Fragmento de cráneo recuperado en LM con coloración rojiza en forma de pequeñas motas. 


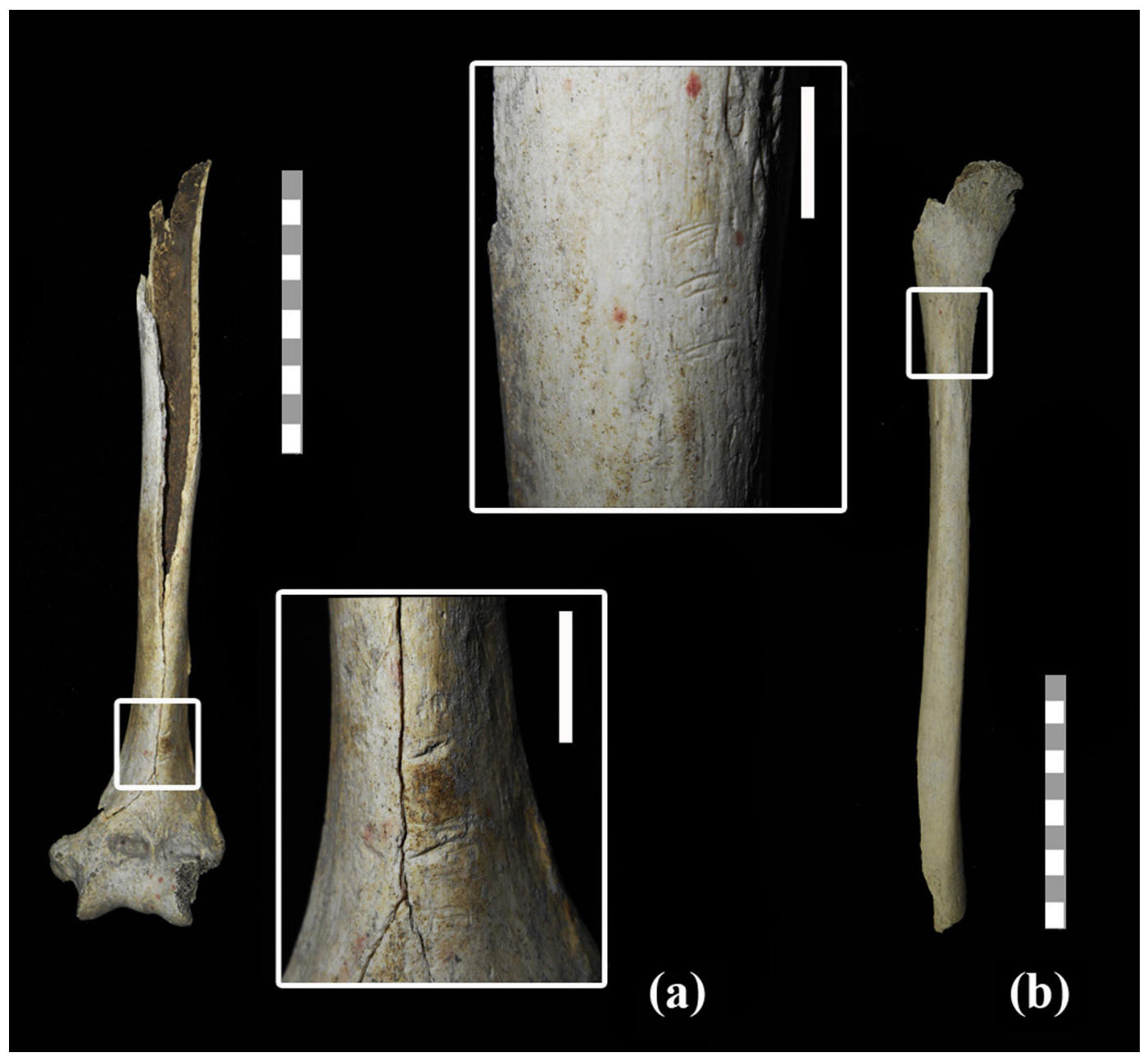

Fig. 4. Evidencias de procesamiento antrópico en elementos de LM. a) Húmero izquierdo con marcas cortas y profundas (vista anterior). b) Tibia izquierda con incisiones paralelas y subparalelas (vista posterior).

1981). Incluso ciertos roedores de hábitos cavadores (e.g. Ctenomys sp.) pueden alterar en sentido vertical y horizontal la distribución espacial de los restos (González, 2009). Observaciones efectuadas en roedores modernos sugieren que los huesos más pequeños son los que poseen mayor probabilidad de ser desplazados por la actividad de estos animales (Bocek, 1986; Davis, Ramsey y Arendale Jr., 1938). Sin embargo, en ninguna de las muestras se han observado marcas que permitan plantear la acción de carnívoros y, en el caso de los roedores, las frecuencias son muy bajas. De todos modos, es posible que los roedores se comporten como agentes más activos en cuanto al desplazamiento de los restos, que dejando marcas de mordidas sobre los mismos
(González, 2009). Respecto de esto, es interesante puntualizar el caso de Fa, el cual registra la mayor cantidad de ausencias óseas, bajas frecuencias de marcas de roedor pero, durante su rescate, se observó una gran cantidad de madrigueras (E. Mange, comunicación personal 2017). Considerando lo expuesto puede plantearse que la marcada escasez de los huesos más pequeños que se registra en las tres muestras podría deberse, en parte, a la acción de los distintos tipos de roedores presentes en la zona del valle (Fernández, Del Papa, Moreira, Prates y De Santis, 2011).

También es posible plantear que las ausencias y bajas representatividades podrían deberse a motivos antrópicos deliberados, tales como la conformación de entierros secundarios. No obstante, 
la evidencia disponible, no indica una clara presencia de entierros secundarios en estos sitios. La única excepción es la del sitio LM, en el cual se registraron algunos elementos óseos con huellas de procesamiento, lo cual podría sugerir presencia de entierros secundarios. Además, aunque los entierros secundarios pueden implicar una selección de partes para conformar los paquetes (Scabuzzo y Politis, 2010), es posible que todos los elementos óseos estén representados, aunque en distintas frecuencias (Martínez, Flensborg y Bayala, 2012). Por último, no puede dejar de mencionarse el efecto antrópico moderno sobre los sitios en términos del deterioro y de la disgregación de los restos. Estos contextos altamente impactados al momento del rescate, sumado a una considerable frecuencia de fracturas en los tres sitios $(>50 \%)$, permiten atribuirle a la acción de la maquinaria un rol preponderante en la ausencia de partes esqueletales. Particularmente, LM es el que mayor impacto sufrió evidenciado por los altos porcentajes de fractura, meteorización y blanqueamiento (Tabla 1). En este caso, la acción de la maquinaria habría sido más intensa que en los otros sitios, resultando en una mayor perturbación del registro, no solo generando un gran deterioro sino también exponiendo y re-exponiéndolo continuamente a los agentes meteorológicos.

Respecto de las depositaciones químicas, en todas las muestras predomina el carbonato de calcio en forma de una delgada película de distribución no homogénea ( $>85 \%$ ). El óxido de manganeso se presenta en forma de pequeñas motas y es poco frecuente en todas las muestras $(<15 \%)$, a excepción del sitio NM3 en donde se registran valores considerablemente mayores (Tabla 1). Independientemente de sus frecuencias, ambos tipos de depositaciones ya han sido documentadas sobre restos humanos procedentes del valle medio en restos humanos (Prates et al., 2011). Además, se registraron rastros de coloración rojiza en las muestras de Fa (1\%) y LM (46\%). En Fa la coloración se presenta como una franja en el sector interno de un borde de fractura de un cráneo, cuyo tejido trabecular retiene rastros de sedimento rojizo (Fig. 3a), lo que indica que la depositación fue posterior a la fractura y al blanqueamiento del espécimen. Es probable que en algún momento de su historia tafonómica, haya estado semi-enterrado y en contacto con alguna concreción rica en óxido de hierro, cuyo origen (natural o antrópico) no puede determinarse con la evidencia disponible. A diferencia del caso anterior, en todos los especímenes de LM la coloración rojiza se observa en forma de motas localizadas (46\%), incorporadas a la matriz ósea (i.e. no superficiales) y altamente asociadas a especímenes blanqueados $\left(\chi^{2}=8.42\right.$, $p<0.01$ ) (Fig. 3b). Aunque no pudo determinarse la composición y origen de estas tinciones, el carbonato de manganeso podría precipitarse y mostrar tonalidades rojizas similares al óxido de hierro (Dupras y Schultz, 2013).

Entre otros procesos que afectaron la superficie ósea, es destacable el impacto de las de raíces sobre los especímenes de NM3 ( $\geq 99 \%$ ), junto con la presencia de deterioro químico. Es probable que este último constituya un efecto de las raíces, lo que sugiere que los restos probablemente se encontraron en un ambiente relativamente estable que permitió el desarrollo de vegetación (Gutiérrez, 2004). Finalmente, se registraron también bajas frecuencias de marcas de roedor sobre los restos de los tres sitios (Tabla 1). La elevada frecuencia de las marcas de raíces, así como la baja para los roedores, son concordantes con las tendencias observadas en otros sitios cercanos del valle del río Negro (Prates et al., 2010b; Prates et al., 2011).

\section{Paleodemografía}

La representación de por lo menos tres individuos adultos en Fa representa la primera evidencia de prácticas mortuorias prehistóricas en el interior de la isla grande de Choele Choel. Si bien aún no se dispone de una cronología absoluta para el sitio, el hecho de que los restos se encontraban depositados sobre la planicie aluvial moderna del río, implica que estos quedarían enmarcados dentro del Holoceno tardío (véase Luchsinger, 2009). En el caso del sitio NM3, las cuantificaciones basadas únicamente en los restos articulados in situ (Serna, 2014), indicaron un número mínimo de cinco adultos de ambos sexos. El análisis aquí realizado suma un total de 7 nuevos individuos (adultos y subadultos) (Tabla 1), elevando el NMI a 12 y ampliando la diversidad etaria de la muestra. Además, el análisis de estos restos permitió mejorar la completitud de uno de los entierros primarios hallados en el sitio (individuo 4). En el caso del sitio $\mathrm{LM}$, dos trabajos previos caracterizaron el número mínimo de individuos, primero de 5 (Prates et al., 
2010b) y luego de aproximadamente 10 (Prates y Di Prado, 2013). Los nuevos resultados precisan y constatan esta última estimación, definiendo un NMI de 10 individuos.

En términos paleodemográficos los resultados permitieron, en primer lugar, incorporar un nuevo sitio $(\mathrm{Fa})$ con evidencias de prácticas mortuorias al valle medio del río Negro, en un sector donde no existían registros previos de este tipo de evidencias. En segundo lugar, aumentar el número de inhumaciones registradas en la zona, así como el espectro etario representado, sumando un total de 15 nuevos individuos. La mayor cantidad de adultos $(n=11$; Tabla 1$)$ en relación a los subadultos ( $n=4$; Tabla 1$)$ contabilizados a partir de las tres muestras, es concordante con lo observado en perfiles paleodemográficos construidos para Patagonia (Suby, Luna, Aranda y Flensborg, 2017). El hecho de contar con un mayor número de elementos e individuos generado a partir de muestras rescatadas, demuestra su potencial para contribuir a la construcción de perfiles de mortalidad de mayor representación para el noreste patagónico (Flensborg, Martínez y Bayala, 2015). Además, la incorporación de estos nuevos resultados al conjunto de datos existentes para el área (véase Prates y Di Prado, 2013), posibilita el planteo de problemas bioarqueológicos a escala poblacional.

\section{Modificación cultural craneana y prácti- cas mortuorias}

A partir de la muestra de LM, se registraron en dos cráneos evidencias de modificación morfológica cultural de naturaleza frontooccipital (Serna, Salazar-García, Valenzuela y Prates, 2017). El cráneo A es compatible con el tipo Tabular Oblicuo (Dembo e Imbelloni, 1938), el cual ya ha sido registrado en LM con un fechado de ca. 2000 años AP (Prates et al., 2010b). Este hallazgo se encuentra dentro de las expectativas espaciales y temporales para esta morfología, que la sitúan en el noreste de Patagonia y sudeste de la región pampeana en un período comprendido entre los ca. 26002000 años AP (Bernal, Gonzalez, Perez y Pucciarelli, 2008; Berón y Baffi, 2003; Madrid y Barrientos, 2000; Perez et al., 2009; Serna et al., 2017; véase también Serna y Prates, 2012). En el cráneo $\mathrm{B}$, aunque se encuentra incompleto, la presencia leve de un "surco transverso frontoparietal" (sensu Dembo e Imbelloni, 1938) o "retrocoronal" (sensu Bórmida, 1953-54) además de una plano lámbdico, plantean la posibilidad de asignarlo a la variante Pseudocircular (Dembo e Imbelloni, 1938). A pesar de que esta variante fue definida como exclusiva de la zona de la laguna del Juncal cerca de la desembocadura del río Negro (Bórmida, 1953-54; Imbelloni, 1924-25), investigaciones recientes señalan su presencia en el noroeste de Patagonia (Della Negra et al., 2014; Perez et al., 2009).

En esta misma muestra, se registraron alteraciones vinculadas con la manipulación de cadáveres, tales como huellas de corte (Fig. 4b) y otras de tipo "chopmarks" (Fig. 4a), atribuibles a la desarticulación (González, 2010; Lyman, 1987; Perez et al., 2005). Hasta este momento en LM sólo se habían registrado inhumaciones de tipo primario y sin ningún tipo de evidencias de procesamiento post o perimortem (Prates et al., 2010a, b). La intervención o manipulación de los cadáveres mediante acciones de procesamiento y/o aplicación de colorantes ha sido frecuentemente documentada en distintos sectores del noreste patagónico (Bórmida, 1950; Favier Dubois et al., 2009; Fisher y Nacuzzi, 1992; Flensborg, Martínez, González y Bayala, 2011; González,2013;MartínezyFiguereroTorres,2000; Martínez, Bayala, Flensborg y López, 2006; Martínez et al., 2014; Moldes de Entraigas, 1983; Mariano, 2011; Moreno, 1874; Outes, 1926; Prates et al., 2011; Peronja et al., 1987; Vignati, 1931). La información cronológica disponible para la región sitúa a este tipo de actividades durante del Holoceno tardío final (Martínez et al., 2014), a diferencia de la cronología estimada para LM en base a entierros primarios, la cual es más temprana (Prates et al., 2010b). Esta discordancia entre la tendencia registrada y la cronología del sitio puede significar que las huellas detectadas representan un caso de procesamiento temprano para el área o, de otro modo, que son el correlato de uno o varios eventos tardíos. Dado que la cuenca del río Negro ha demostrado ser un sector del paisaje de uso recurrente durante el Holoceno tardío (Prates y Di Prado, 2013), es razonable pensar que en LM se inhumaron individuos en forma primaria durante la parte inicial y con algún grado de intervención antrópica hacia finales de este período. 


\section{Consideraciones finales}

Del estudio del registro altamente perturbado de los tres sitios se desprenden varios puntos de interés. En primer lugar, se incorporó un nuevo sitio con inhumaciones $(\mathrm{Fa})$ en la isla grande de Choele Choel, además de ampliarse y ajustarse el número mínimo de individuos y la representación etaria pre-existente para otros dos sitios (NM3 y LM) del valle medio del río Negro. En segundo lugar, la presencia de dos nuevos cráneos deformados en LM significa, en el caso del Tabular Oblicuo, un nuevo registro de una modificación poco frecuente $y$, en el caso del posible Pseudocircular, una ampliación en su esquema de distribución espacial original (Dembo e Imbelloni, 1938). Finalmente, el hallazgo de huellas de procesamiento sobre restos humanos en LM amplía la variabilidad y complejidad de la conducta mortuoria desplegada en el sitio.

En suma, el registro bioarqueológico altamente impactado por la acción moderna ha demostrado ser un referente empírico útil y válido para ser incorporado a la discusión arqueológica local y regional. En la actualidad la bioarqueología y la antropología biológica se encuentran inmersas en un momento de intenso diálogo con las comunidades descendientes de pueblos originarios y cumpliendo un rol central en sus reivindicaciones (Ametrano, 2015; Aranda, Barrientos y Del Papa, 2014; Endere, 2011; Guichón et al., 2015; Sardi, Reca y Pucciarelli, 2015; Sardi, 2016). En esta coyuntura histórica se pone en evidencia, más que nunca, la importancia y necesidad de ponderar el registro perturbado tomando acciones de rescate y conservación que impidan su destrucción y que permitan su estudio científico y valoración social en un marco de entendimiento mutuo y respeto (Aranda et al., 2016).

\section{AGRADECIMIENTOS}

Este trabajo fue posible gracias a los subsidios otorgados por el PICT 2015-3645. A Luciano Prates y Emiliano Mange por su lectura crítica del manuscrito. A los dos evaluadores anónimos quienes contribuyeron a mejorar substancialmente este trabajo con sus comentarios y sugerencias.

\section{LITERATURA CITADA}

Adams, B. J. y Byrd, J. E. (2008). Recovery, analysis, and identification of commingled human remains. Nueva York, Estados Unidos: Springer Science \& Business Media. doi: 10.1007/978-1-59745-316-5

Ametrano, S. J. (2015). Los procesos de restitución en el Museo de La Plata. Revista Argentina de Antropología Biológica, 17(2), 1-13. doi: 10.17139/ raab.2015.0017.02.03

Aranda, C., Barrientos, G. y Del Papa M. C. (2014). Código deontológico para el estudio, conservación y gestión de restos humanos de poblaciones del y pasado. Revista Argentina de Antropología Biológica, 16(2), 111-113. doi: 10.17139/raab.2014.0016.02.05

Aranda, C., Luna, L., Costanzo, N., Contissa, V., Natalia, G., Godoy, P. y Zuccala, K. (2016). Conservación e investigación en el área de Antropología Biológica del $\mathrm{Mu}-$ seo Etnográfico JB Ambrosetti (Facultad de Filosofía y Letras, Universidad de Buenos Aires, Argentina). Antropologia Portuguesa, 30, 11-28. doi: 10.14195/21827982_31_1

Behrensmeyer, A. K. (1978). Taphonomic and ecological information from bone weathering. Paleobiology, 4,(2), 150-162. doi: 10.1017/S0094837300005820

Bernal, V., Gonzalez, P. N., Perez, S. I y Pucciarelli, H. M. (2008). Entierros humanos del noreste de Patagonia: nuevos fechados radiocarbónicos. Magallania, 36(2), 175-183. doi: 10.4067/S0718-22442008000200013

Berner, R. A. (1981). Authigenic mineral formation resulting from organic matter decomposition in modern sediments. Fortsher Miner, 59(1), 117-135.

Berón, M. A. y Baffi, E. I. (2003). Procesos de cambio cultural en los cazadores-recolectores de la provincia de La Pampa, Argentina. Intersecciones en Antropología, 4, 29-43.

Binford, L. R. (1981). Bones: ancient men and modern myths. Nueva York, Estados Unidos: Academic Press.

Bocek, B. (1986). Rodent ecology and burrowing behavior: predicted effects on archaeological site formation. American Antiquity, 51(3), 589-603. doi: 10.2307/281754.

Borella, F., Mariano, C. y Favier Dubois, C. (2007). Procesos tafonómicos en restos humanos en superficie en la localidad arqueológica de Bajo de la Quinta, Golfo San Matías (Río Negro). En Morello, F., Martinic, M., Prieto, A. y Bahamonde G. (Eds.), Levantando piedras, desenterrando huesos... y develando arcanos (pp. 403410). Punta Arenas, Chile: Ediciones CEQUA.

Bórmida, M. (1950). Cementerios indígenas prehispánicos en la zona de la Laguna del Juncal. Anales del Museo Nahuel Huapi, 2, 101-108.

Bórmida, M. (1953-54). Los antiguos Patagones. Estudio de craneología. Runa, 6(1-2), 55-96.

Buikstra, J. y Ubelaker, D. (1994). Standards for data collection from human skeletal remains. Faytteville, Estados Unidos: Arkansas Archaeological Survey Research Series No. 44.

Cocilovo, J. A. y Guichón, R. A. (1994). La deformación craneana "pseudocircular" en el grupo prehistórico de Laguna del Juncal. Antropología Biológica, 2(2), 13-27.

Davis, W. B., Ramsey, R. R. y Arendale Jr, J. (1938). Distribution of pocket gophers (Geomys breviceps) in relation to soils. Journal of Mammalogy, 19, 412-418. doi:10.2307/1374224

Della Negra, C., Novellino, P., Gordón, F., Vázquez, R., Béguelin, M., González, P. y Bernal, V. (2014). Áreas de entierro en cazadores-recolectores del Noroeste de 
Patagonia: sitio Hermanos Lazcano (Chos Malal, Neuquén). Runa 35(2), 5-19.

Del Papa, M., Gordón, F., Castro, J. C., Fuchs, L., Menéndez, L., Di Bastiano, A., Pucciarelli, H. M. (2011). Un cráneo del norte de Patagonia con modificaciones postmórtem: Aproximación mediante técnicas no invasivas. Intersecciones en Antropología, 12(2),349-354.

Dembo, A. e Imbelloni, J. (1938). Deformaciones intencionales del cráneo de carácter étnico. Buenos Aires, Argentina: J. Anesi.

Dupras, T. L, y Schultz, J. J. (2013). Taphonomic bone staining and color changes in forensic contexts. Boca Raton, Estados Unidos: CRC Press. doi:10.1201/b15424-13

Endere, M. L. (2011). Cacique Inakayal. La primera restitución de restos humanos ordenada por ley. Corpus. Archivos virtuales de la alteridad americana, 1(1). doi:10.4000/corpusarchivos.937

Favier Dubois, C. M., Borella, F. y Tykot, R. (2009). Explorando tendencias temporales en el uso humano del espacio y los recursos marinos en el Golfo San Matías (Río Negro). En Salemme, M., Santiago, .F, Alvarez, M., Piana, E., Vázquez, M. y Mansur, E. (Eds.). Arqueología de la Patagonia: una mirada desde el último confin (pp.985-997). Ushuaia, Argentina: Editorial Utopías.

Fernández, F. J., Del Papa, L. M., Moreira, G. J., Prates, L. y De Santis, L. J. (2011). Small mammal remains recovered from two archaeological sites in the middle and lower Negro River valley (Late Holocene, Argentina): Taphonomic issues and paleoenvironmental implications. Quaternary International, 245(1), 136-147. doi: 10.1016/j.quaint.2010.12.027

Fisher, A. y Nacuzzi, L. R. (1992). La destrucción sistemática del paisaje y de los sitios arqueológicos. El caso del Valle de Viedma. Arqueología, 2, 189-229.

Flensborg, G., Martínez, G., González, M. y Bayala, P. D. (2011). Revisión de los restos óseos humanos del sitio La Petrona (Transición Pampeano-Patagónica oriental, Argentina). Magallania, 39(1), 179-191. doi:10.4067/ S0718-22442011000100011

Flensborg, G., Martínez, G. y Bayala, P. D. (2015). Mortality profiles of hunter-gatherer societies: a case study from the eastern Pampa-Patagonia transition (Argentina) during the final late holocene. International Journal of Osteoarchaeology, 25(6), 816-826. doi:10.1002/oa.2348

González ME. (2009). Análisis de los efectos tafonómicos en los restos óseos humanos de Laguna Tres Reyes 1: la actividad perturbadora de los roedores. En Bourlot, T., Bozzuto, D., Crespo, C., Hetch, A. C. y Kuperszmit, N. (Eds.), Entre pasados y presentes II. Estudios contemporáneos en Ciencias Antropológicas (pp.307-322). Buenos Aires, Argentina: Fundación de Historia Natural Félix de Azara.

González ME. (2010). Huellas de corte y análisis contextual en restos óseos humanos de la cuenca inferior del río Colorado: implicaciones para el entendimiento de las prácticas mortuorias. En Berón, M., Luna, L., Bonomo, M., Montalvo, C., Aranda, C. y Carrera Aizpitarte, M. (Eds.), Mamul Mapü: pasado y presente. Perspectivas de la arqueología pampeana a comienzos del tercer milenio (pp. 193-210). Buenos Aires, Argentina: Liibros del Espinillo.

González, M. E. (2013). Procesos de formación y efectos tafonómicos en entierros humanos: el caso del sitio Paso Alsina 1 en Patagonia nororiental Argentina. Magallania, 41(1), 133-154. doi:10.4067/S071822442013000100007

Grayson, D. K. (1984). Quantitative zooarchaeology. Nueva York, Estados Unidos: Academic Press.

Gutiérrez, M. A. (2004). Análisis tafonómicos en el área interserrana (Provincia de Buenos Aires). Tesis de doctorado. Facultad de Ciencias Naturales y Museo, Universidad Nacional de La Plata, La Plata.

Guichón, R.A., García Laborde, P., Motti, J., Martucci, M., Casali, R., Huilinao, F. y Gallardo Pantoja, C. (2015). Experiencias de trabajo conjunto entre investigadores y pueblos originarios. Revista Argentina de Antropología Biológica, 17(2), 1-8. doi: 10.17139/ raab.2015.0017.02.05

Guichón, R. A., Muñoz, A. S. y Borrero, L. A. (2000). Datos para una tafonomía de restos óseos humanos en Bahía San Sebastián, Tierra del Fuego. Relaciones de la Sociedad Argentina de Antropología, 25, 297-311.

Imbelloni, J. (1924-25). Deformaciones intencionales del cráneo en Sudamérica; polígonos craneanos aberrantes. Revista del Museo de La Plata, 28, 329-407.

Knüsel, C. J. y Outram, A. K. (2004). Fragmentation: The zonation method applied to fragmented human remains from archaeological and forensic contexts. Environmental Archaeology, 9(1), 85-98. doi:10.1179/ env.2004.9.1.85

Luchsinger, H. M. (2009). The late Quaternary landscape history of the middle rio Negro valley, Northern Patagonia, Argentina: Its impact on preservation of the archaeological record and influence on Late Holocene human settlement patterns. Tesis de doctorado. A\&M Texas University, College Station, Texas.

Luna, L. H. (2010). Alternative methodological procedures in sex determination of commingled and fragmentary human remains: an example from Argentine Pampean region. En Weiss, K. (Ed.), Trends in physical anthropology (pp. 1-48). Nueva York, Estados Unidos: Nova Science Publishers, Inc.

Luna, L. H. y Aranda, C. M. (2005). Evaluación de marcadores sexuales de individuos subadultos procedentes del sitio Chenque I (Parque Nacional Lihué Calel, provincia de La Pampa, República Argentina). Revista Española de Antropología Física, 25, 25-40.

Lyman, R. L. (1987). Archaeofaunas and butchery studies: a taphonomic perspective. Advances in archaeological method and theory, 10, 249-337. doi:10.1016/B978-012-003110-8.50008-6

Lyman, R. L. (1994). Vertebrate taphonomy. Cambridge, Reino Unido: Cambridge University Press. doi:10.1002/ gea.3340110306

Lyman, R. L. (2008). Quantitative paleozoology (Cambridge manuals in archaeology). Cambridge, Reino Unido: Cambridge University Press. doi:10.1017/ CBO9780511813863

Lyman, R. L. (2010). What taphonomy is, what it isn't, and why taphonomists should care about the difference. $\mathrm{JO}_{\mathrm{O}}$ urnal of Taphonomy, 8(1), 1-16.

Madrid, P. y Barrientos, G. (2000). La estructura del registro arqueológico del sitio Laguna Tres Reyes 1 (Provincia de Buenos Aires): Nuevos datos para la interpretación del poblamiento humano del Sudeste de la región Pampeana a inicios del Holoceno tardío. Relaciones de la Sociedad Argentina de Antropología, XXV, 179-206.

Mange, E., Prates, L., González Venanzi, L. y Di Lorenzo, M. (2016). El registro faunístico del sitio Negro Muerto 3 (provincia de río Negro, argentina): tafonomía y patrones de explotación. Comechingonia, 20(1), 231-252.

Mariano, C. I. (2011). Prácticas mortuorias y registro bioarqueológico en la costa rionegrina del golfo San Matías, Argentina. Intersecciones en Antropología, 12(1), 17-30.

Martin, F. M., Barberena, R. y Guichón, R. A. (2004). Erosión y huesos humanos. El caso de la localidad Chorrillos, Tierra del Fuego. Magallania, 32, 125-142. 
Martínez, G. (2010). Entierros humanos en lugares sagrados y domésticos durante el Holoceno Tardío: el registro bioarqueológico del curso inferior del Río Colorado (provincia de Buenos Aires, Argentina). Werkén, 13, 145-160.

Martínez, G., Bayala. P. D, Flensborg, G. y López, R. (2006). Análisis preliminar de los entierros humanos del sitio Paso Alsina 1 (Pcia. de Buenos Aires). Intersecciones en Antropología, 7, 95-108.

Martínez, G. y Figuerrero Torres, MJ. (2000). Sitio arqueológico La Petrona (partido de Villarino, Provincia de Buenos Aires): Análisis de las modalidades de entierro en el área sur pampeana. Relaciones de la Sociedad Argentina de Antropología, 25, 227-247.

Martínez, G., Flensborg, G. y Bayala, P. D. (2012). Human corpse manipulation and the body as symbol: A case study from the Eastern Pampa-Patagonia transition (Argentina) during the Final Late Holocene. Journal of Anthropological Archaeology, 31(2), 215-226. doi:10.1016/j.jaa.2011.12.002

Martínez, G., Martínez, G. A., Stoessel, L., Alcaráz, A. P., Valero, F. S., Flensborg, G. y Onorato, J. F. (2014). Resultados preliminares del sitio Zoko Andi 1. Aportes para la arqueología del curso inferior del río Colorado (Provincia de Buenos Aires). Revista del Museo de Antropología, 7(1), 105-118.

Moldes de Entraigas, B. (1983). Arqueología y etnohistoria del bajo curso del Río Negro. Presencia hispánica en la arqueología Argentina, 2, 877-893.

Moreno, F. P. (1874). Description des cimetières et paraderos prehistoriques de patagognie. Revue d'Antropologie, 3,72-90.

Outes, F. (1926). Noticias sobre el resultado de mis investigaciones antropológicas en la extremidad sudeste de la Provincia de Buenos Aires. Physis, 8, 387-390.

Perez, S. I., Della Negra, C., Novellino, P., Gonzalez, P. N., Bernal, V., Cuneo, E. y Hajduk, A. (2009). Deformaciones artificiales del cráneo en cazadores recolectores del Holoceno medio-tardío del noroeste de Patagonia. Magallania, 37(2), 77-90. doi:10.4067/S071822442009000200005

Perez, V. R., Godfrey, L. R., Nowak-Kemp, M., Burney, D. A., Ratsimbazafy, J. y Vasey, N. (2005). Evidence of early butchery of giant lemurs in Madagascar. Journal of Human Evolution, 49(6), 722-742. doi:10.1016/j.jhevol.2005.08.004

Peronja, A., Sánchez y Juliá, E. y López, A. (1987). Prospecciones arqueológicas sobre costa de río, con presencia de bivalvos, Provincia de Río Negro. Actas de las Primeras Jornadas de Arqueología de Patagonia (pp. 243-248). Serie Humanidades. Gobierno de la provincia de Chubut, Chubut.

Prates, L. y Di Prado, V. (2013). Sitios con entierros humanos y ocupaciones residenciales en la cuenca del Río Negro (Norpatagonia, Argentina): diacronía y multicausalidad. Latin American Antiquity, 24(4), 451-466. doi:10.7183/1045-6635.24.4.451

Prates, L., Di Prado, V., Mange, E. y Serna, A. (2010a). Sitio arqueológico Loma de los Muertos. Múltiples ocupaciones sobre un médano del este de Norpatagonia (Río Negro, Argentina). Magallania, 38(1), $163-$ 179. doi:10.4067/S0718-22442010000100010

Prates, L., Flensborg, G. y Bayala, P. D. (2010b). Caracterización de los entierros humanos del sitio Loma de los Muertos (valle medio del Río Negro, Argentina). Magallania, 38(1), 147-162. doi: 10.4067/S071822442010000100009

Prates, L., Luchsinger, H., Scabuzzo, C, y Mansegosa, D. (2011). Arqueología y bioarqueología del sitio La Vic- toria 5 (Departamento de General Conesa, Río Negro). Intersecciones en Antropología, 12, 155-167.

Prates, L. y Serna, A. (2015). Cuantificación de los restos óseos retirados por la policía en el barrio Cooperativa 8 de Diciembre (Viedma, Pcia. de Río Negro). Informe disponible en la Secretaria de Cultura de la Provincia de Río Negro y en el Lab. $N^{\circ} 3$ de la División de Arqueología del Museo de La Plata.

Prates, L., Serna, A., Mange, E. y de Jong, I. (2016). Expresión material de la interacción entre indígenas y criollos en un sitio funerario del siglo XIX de Norpatagonia (Sitio Chimpay, Río Negro). Intersecciones en Antropología, 17, 35-48.

Sardi, M. (2016). Solicitud de restitución de los restos de Manuel Guerra, general, indio brujo y chipitruz. Informe de las secciones "Colecciones. Documentación y Registro" y "Demandas académicas y comunitarias". División Antropología, Museo de La Plata. División Antropología, Museo de La Plata. Referencia Expte. 1000-005362/16-000

Sardi, M.L., Reca, M. M. y Pucciarelli, H. M. (2015). Debates y decisiones políticas en torno de la exhibición de restos humanos en el Museo de La Plata. Revista Argentina de Antropología Biológica, 17(2), 1-8. doi:10.17139/raab.2015.0017.02.04

Scabuzzo, C. y Politis, G. (2011). Entierros secundarios del Holoceno temprano y medio en la región pampeana. Nuevos datos del sitio Arroyo Seco 2. Cazadores Recolectores del Cono Sur 4, 135-155.

Scheuer, L. y Black, S. (2000). Developmental juvenile osteology. Londres, Reino Unido: Academic Press.

Schutkowski, H. (1993). Sex determination of infant and juvenile skeletons: I. Morphognostic features. American Journal of Physical Anthropology, 90, 199-205. doi:10.1002/ajpa.1330900206

Serna, A. (2014) Rescate en la estancia Negro Muerto: el caso del sitio Negro Muerto 3 (provincia de Río Negro, Argentina). Libro de resúmenes de las IX Jornadas de Arqueología de la Patagonia. Chile.

Serna, A. y Prates, L. (2012). Bioarqueología y cronología del sitio Negro Muerto 2 (Noreste de Patagonia). Magallania, 40(2), 233-245. doi:10.4067/S071822442012000200011

Serna, A., Prates, L. y Luna, L. (2015). Osteobiografia de dos individuos inhumados durante la Campaña del Desierto: el caso del sitio Chimpay (Argentina). Revista Española de Antropología Americana, 45(2), 419-437. doi:10.5209/REAA.54934

Serna, A., Salazar-Garcia, D. C., Valenzuela, L. O. y Prates, L. (2017). Movilidad humana en el noreste de la Patagonia durante el Holoceno tardío. Análisis preliminar de isótopos estables de oxígeno. Libro de resúmenes de las X Jornadas de Arqueología de Patagonia. Puerto Madryn, Argentina.

Suby, J., Luna, L., Aranda, C. y Flensborg, G. (2017). First approximation to paleodemography through age-at-death profiles in hunter-gatherers from Southern Patagonia during middle-late Holocene. Quaternary International, 438, 174-188. doi:10.1016/j.quaint.2017.04.035

Ubelaker, D. (1989). The estimation of age at death from immature human bone. En Isçan, M. (Ed.), Age markers in the human skeleton (pp. 55-70). Springfield, Estados Unidos: Ch. C. Thomas Publisher.

Vignati, M. (1931). Investigaciones antropológicas en el litoral marítimo sudatlántico bonaerense. Notas preliminares del Museo de La Plata Tomo I, 19-31.

White, T. y Folkens, P. A. (2005). The human bones manual. Londres, Reino Unido: Elsevier Academic Press. 